\title{
An overview of anesthetic procedures, tools, and techniques in ambulatory care
}

This article was published in the following Dove Press journal:

Ambulatory Anesthesia

27 January 2015

Number of times this article has been viewed

\section{Zakaria Messieha}

Department of Anesthesiology, University of Illinois at Chicago, Chicago, IL, USA

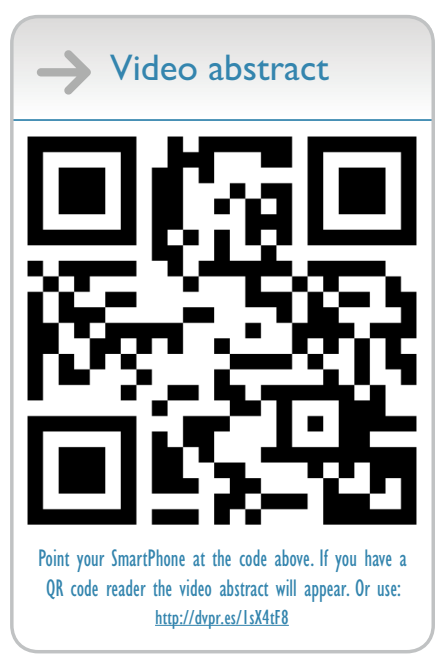

Correspondence: Zakaria Messieha Department of Anesthesiology, University of Illinois at Chicago,

1740 West Taylor Street, Chicago,

IL 606/2-7239, USA

Email messieha@uic.edu
Abstract: Ambulatory surgical and anesthesia care (ASAC), also known as Same Day Surgery or Day Care in some countries, is the fastest growing segment of ambulatory surgical and anesthesia care. Over 50 million ambulatory surgical procedures are conducted annually comprising over $60 \%$ of all anesthesia care with an impressive track record of safety and efficiency. Advances in ambulatory anesthesia care have been due to newer generation of inhalation and intravenous anesthetics as well as airway management technology and techniques. Successful ambulatory anesthesia care relies on patient selection, adequate facilities, highly trained personnel and quality improvement policies and procedures. Favoring one anesthetic technique over the other should be patient and procedure-specific. Effective management of post-operative pain as well as nausea and vomiting are the final pieces in assuring success in ambulatory anesthesia care. Keywords: ambulatory anesthesia, out-patient anesthesia, Day-Care anesthesia

\section{Introduction}

Ambulatory surgical and anesthesia care (ASAC), also known as same-day surgery or day care in some countries, is the fastest-growing segment of surgical and anesthesia care. Over 50 million ambulatory surgical procedures are conducted annually, comprising over $60 \%$ of all anesthesia care, with an impressive track record of safety and efficiency. The economics of health care as well as patient expectations have increased the need for moving more of the surgical and anesthesia care to the ambulatory setting. This article addresses the advances in techniques and the culture of safety of ASAC.

\section{Culture of safety}

In a recently published edition of Anesthesiology Clinics of North America, Fleisher ${ }^{1}$ stated that ambulatory anesthesia is becoming the predominant type of surgical and anesthesia care provided. He stressed that the advances made in this area have been made in multiple domains, including patient comorbidity management, drug development, and regulatory issues, as well as outcome assessment. In the same edition, Apfelbaum and Cutter ${ }^{2}$ discussed the culture of safety through what they termed the "four Ps": place, procedure, personnel, and patient.

Physical facilities where ASAC takes place can vary from a dedicated facility within a hospital, free-standing ambulatory surgical center (ASC) or office-based facility. All three settings can be very safe and appropriate, provided that the required facilities are well equipped and at the level that would be conducive to safe provision of care. Back-up systems as well as advanced airway-management techniques are very important to deal with the unexpected. Apfelbaum and Cutter place great emphasis on 
the presence of highly trained personnel, including surgical, anesthesia, and administrative personnel.

They summarized patient-related challenges in the following questions:

1. Is the patient stable enough to tolerate ASAC?

2. Can postoperative pain be managed effectively after discharge?

3. Is there a significant risk of postoperative nausea and vomiting?

4. Is there a significant risk of postoperative pathophysiologic derangements?

If those risks are significant, then the patient may not be a suitable candidate for ASAC. Weiss et $\mathrm{al}^{3}$ stated that hospital stays involving an operating room procedure were about twice as costly as those that did not involve operating room procedures. Mathis et $\mathrm{al}^{4}$ identified those at high risk for major complications subsequent to ASAC. Of 244,397 surgeries studied, they reported a $0.1 \%$ risk of developing early perioperative morbidity. Seven independent risk factors were identified while controlling for surgical complexity: overweight body mass index, obese body mass index, chronic obstructive pulmonary disease, history of transient ischemic attacks/ stroke, hypertension, previous cardiac surgical intervention, and prolonged operative time. Hollingsworth et $\mathrm{al}^{5}$ reported that the most prevalent ASAC procedures are ophthalmology and plastic surgery. Absent from that study were dental and oral surgery procedures, which if taken into consideration can add to the significance and extent of ambulatory surgical procedures conducted. A study conducted by the University of Michigan published by Sheetz et $\mathrm{al}^{6}$ reported a $2.3 \%$ incidence of postoperative complications for ASAC compared to $7.4 \%$ for inpatient procedures. They attributed the wide variation in utilization of ASAC to variation in surgeon practices.

In an effort to stratify liability risk for ambulatory anesthesia Metzner and $\mathrm{Kent}^{7}$ painted a mixed picture of reduced liability risk of ASAC, reporting a reduced number of claims for major damaging events, yet indicated that adverse events occurring after discharge remain a particular risk for liability, especially for office-based procedures.

When looking outside the US, a national Danish study published by Majholm et $\mathrm{al}^{8}$ reported no deaths in 57,709 ASAC cases, with a $1.21 \%$ incidence of return to hospital. Gynecological, orthopedic, and gastrointestinal procedures were the most prevalent procedures, with postoperative bleeding and infection the most common two reasons for hospital returns.

Nekhendzy and Ramaiah ${ }^{9}$ addressed the prevention of perioperative anesthesia-related complications in facial cosmetic surgery with the following general considerations:
1. accreditation of facility

2. top-down approach to assure compliance with American Society of Anesthesiologists guidelines and presence of established policies and procedures

3. bottom-up approach with quality improvement and personnel training

4. presence of expert anesthesiologist and surgeon with appropriate knowledge and experience in office-based anesthesia

5. a team approach to patient care

6. safety checklists.

While some of the guidelines may not be evidence-based, they underscore the increasing attention toward safety and quality improvement. Perioperative risk-reduction recommendations were summarized as follows:

1. proper patient selection and preparation

2. routine prophylaxis for deep-vein thrombosis and pulmonary embolism

3. special attention to deep-sedation cases

4. preference for rapidly titratable drugs

5. quiet, clear surgical field: maintenance of controlled hypotension

6. absence of patient movement

7. preference for total intravenous anesthesia

8. avoidance of nitrous oxide

9. preference for the use of a laryngeal mask airway (LMA)

10. smooth nonstimulating emergence from anesthesia

11. rapid awakening and return to protective airway reflexes

12. aggressive prevention of postoperative nausea and vomiting (PONV)

13. fast-tracking patients for discharge

14. minimizing doses of intraoperative and postoperative opioids

15. prevention of rebound hypertension

16. familiarity with procedure-specific requirements and complications.

\section{Culture of efficiency}

Looking at 21 nonacademic facilities, Dexter et $\mathrm{al}^{10}$ found that while inpatients scheduled for surgery accounted for $16.2 \%$ of all scheduled surgeries versus same-day surgery patients, they accounted for $49 \%$ of cancelled procedures. All same-day surgery patients had a preanesthesia assessment by phone. They accounted for $1.6 \%$ of the scheduled minutes that were canceled.

With the emergence of free-standing ASC, Hair et al ${ }^{11}$ compared visits of Medicare beneficiaries to hospital- 
based versus free-standing ASC. Their study showed that perioperative time was 39\% shorter in free-standing ASC. Surgery time was $37 \%$ shorter, operating room time was $37 \%$ shorter, and postoperative time was $35 \%$ shorter, hence improving efficiency and cost-effectiveness. Clark et $\mathrm{al}^{12}$ demonstrated similar trends for endocrine procedures conducted in dedicated ASAC hospital operating rooms. This can lead us to conclude that efficiency and cost-effectiveness are two clear advantages of the ambulatory setting and culture.

\section{Office-based anesthesia}

Office-based anesthesia and surgical care is the fastest growing segment of ASAC. The number of office-based procedures increased to 10 million procedures per year from 1995 to $2005 .^{13}$ Safety data vary greatly among different studies, as outlined by Shapiro et al. ${ }^{14,15}$ They indicated that while some authors focused on plastic surgery outcomes, particularly liposuction and abdominoplasty, which might carry a higher risk regardless of duration of surgery, which seems not to be a contributing factor, ${ }^{16}$ other studies looked at office-based procedures across the board and found low complications during these procedures, with evidence of morbidity and mortality profiles similar to those of other ambulatory settings. As is the case with any other setting, the safety criteria and practices that were outlined earlier should be adhered to in order to reduce the risk of adverse outcomes. An emphasis on continuous quality improvement, safety checklists, and outcome data reporting is very important to ensure high levels of safety and quality while fostering a culture of efficiency and cost-effectiveness.

\section{Airway management}

Significant advances in airway-management technology and anesthetic quality and safety have taken place in the past 25 years. In this segment, some of those advancements with regard to safe airway management will be outlined.

\section{Laryngeal mask airway}

The LMA is a supraglottic device that facilitates airway ventilation without tracheal intubation. It is inserted primarily through a blind technique, with some variations depending on the type of LMA being utilized. There are several designs that have evolved since the initial introduction of the LMA into clinical use that can facilitate ventilation and serve as conduits in tracheal intubation and for suction of secretion, as well as improve the airway seal to minimize intrapulmonary aspiration. Use of the LMA in ambulatory anesthesia is common, and has been proven to be safe and effective. ${ }^{17}$ In ambulatory pediatric ophthalmologic procedures, a retrospective study showed that the insertion of an LMA without the need of intravenous access was both safe and effective. ${ }^{18}$ ProSeal LMA was found to create a better seal from gastric aspiration, but was more challenging to insert. ${ }^{19}$ The selection of the type of LMA for use should be procedure- and patient-dependent and at the discretion of the anesthesiologist. In general, LMA utilization has the following limitations:

1. limited efficacy in delivering high peak airway pressures

2. carries a certain risk of intrapulmonary aspiration

3. may interfere with certain procedures, particularly intraoral and laryngeal procedures.

\section{Video-assisted laryngoscopy}

Videolaryngoscopy emerged into clinical use over 10 years ago. It has provided an added level of sophistication to advanced airway management. Studies have demonstrated a higher success rate with videolaryngoscopy versus conventional direct laryngoscopy. ${ }^{20-22}$ This provides a significant advantage in the ambulatory setting in efforts to improve quality of care and minimize airway trauma or intubation failure. Multiple designs of video-assisted laryngoscopes have emerged, including compact designs that can be quite valuable in office-based and mobile anesthesiology settings. As in any other techniques, limitations pertain to technical difficulties, fluid and blood contamination of the camera, and failure to visualize the glottis.

\section{Flexible-tip endotracheal tubes}

In an effort to improve quality of care and minimize airway trauma, flexible-tip endotracheal tubes offer considerable promise. A flexible tip that has been incorporated in the manufacturing process of these endotracheal tubes offers a less traumatic alternative during nasal and oral endotracheal intubation. ${ }^{23-25}$ Flexible-tip endotracheal tubes require some adaptation when conducting a nasal intubation technique, and might require some practice before the provider is fully proficient in utilizing them, but they can be useful tools in the effort to minimize airway-intubation trauma. More double-blind studies are needed to confirm their advantage over conventional endotracheal tubes.

\section{Advancements in anesthetic techniques}

An ideal anesthesia technique is one that has fast onset and fast recovery, with minimal cardiovascular and side effects. While science is still in search of the holy grail of anesthesia care, many advances have been made in the past two decades 
that have improved safety and outcomes of anesthetic techniques. This segment will outline some of the current anesthetics that have made a significant impact on the safety of ambulatory anesthesia techniques.

\section{Inhalation anesthesia}

Volatile inhaled anesthetics have been the backbone of surgical anesthesia care. Several agents have been developed since the 1950s that have significantly improved the safety track record of this class of drugs. The introduction of sevoflurane and desflurane as part of patient care in the 1990s further improved patient-safety outcomes, especially in the areas of induction and recovery.

\section{Sevoflurane}

Sevoflurane provides several advantages as a mask induction agent in comparison with halothane, which had been used for such purposes for several decades. While both agents provide minimal upper-airway irritability, sevoflurane has far fewer cardiovascular suppressive effects and lesser risk of cardiac arrhythmia. Sevoflurane has a blood-gas partition coefficient of 0.69 and blood-brain coefficient of approximately 1.7 , which is significantly lower than the older-generation volatile anesthetics, thus facilitating faster induction and faster recovery. Reduction of systemic vascular resistance is dose-dependent and comparable to other agents. As a potent bronchodilator, sevoflurane is a safe agent for individuals with asthma and reactive airway disease. Compared to halothane, sevoflurane has a significantly higher incidence of delirium and agitation in children ${ }^{26}$ requiring intervention to improve the quality of recovery.

\section{Desflurane}

With a blood-gas partition coefficient of approximately 0.42 and brain-blood partition coefficient of approximately 1.3, desflurane has demonstrated significantly faster recovery characteristics with minimal redistribution, making it the inhalation agent of choice for the obese patient. Being a highly irritant agent to the upper airway, desflurane is not an agent of choice for mask induction, due to serious concerns with inducing laryngospasm. Its boiling point at room temperature requires the need for a special vaporizer for administration of desflurane.

All volatile anesthetics are trigger agents for malignant hyperthermia, requiring the presence of dantrolene sodium in the facility, and are an absolute contraindication for patients with such history. Both sevoflurane and desflurane come at a much higher cost compared to isoflurane and halothane.

With those two newer agents offering several advantages over the older-generation anesthetics, the verdict is not completely out on which is more advantageous compared to the other. White et $\mathrm{al}^{27}$ evaluated 130 patients undergoing superficial outpatient surgeries. All patients were induced with propofol and maintained with either sevoflurane or desflurane with an LMA as the airway of choice. The desflurane group had $60 \%$ incidence of coughing compare to $32 \%$ for sevoflurane. Emergence from anesthesia was more rapid in the desflurane group, however, in the late recovery period there was no significant difference between the two groups.

In a smaller study involving adults undergoing a laryngoscopy procedure, Nathanson et $\mathrm{al}^{28}$ reported similar outcomes compared to the White et al study, with similar outcomes regarding PONV and patient acceptance of the anesthetic for future procedures. It is worth mentioning that a metaanalysis study published by Dexter and Tinker $^{29}$ showed a mean discharge time for desflurane group only 4.4 minutes faster than the isoflurane group, while the propofol group was 17 minutes faster than the desflurane group.

\section{Intravenous anesthetics}

There is a growing trend in ASAC for utilization of intravenous anesthetics as the sole technique for provision of ambulatory anesthesia care, also known as total intravenous anesthesia. This came about as a result of the advancement in pharmacokinetics and dynamics of injectable anesthetic agents, as well as the better clinical outcomes of several of those anesthetics. This segment will address the impact of several newer intravenous anesthetics on the evolution of ambulatory anesthesia. It is important for the anesthesia provider not to be locked in a single technique, since patient needs and circumstances vary, and often different techniques, as well as hybrid techniques, between inhalation and intravenous anesthetics may be the best approach in a particular situation.

\section{Propofol}

Propofol was initially developed in Europe in the late 1970s, and was then removed from the market, due to anaphylactic reactions related to the vehicle solution. It was reintroduced in the mid 1980s, and has become the most commonly utilized intravenous induction agent. Propofol is a diisopropyl anesthetic agent with soy oil and egg phospholipid as part of the preparation. Favorable clinical effects include rapid onset and recovery, as well as potent amnestic effects and reduction of PONV. Propofol should be used with caution in patients with significant cardiac failure, due to its possible depressive effect on cardiac output and systemic vascular resistance. Propofol can be utilized as a single-dose induction agent and for continuous infusion at general anesthesia and deep-sedation levels, as 
well as in combination with other intravenous agents. Concerns regarding soy allergies and egg-lecithin allergies should be taken into consideration when using propofol.

Fospropofol is a water soluble prodrug of propofol that was introduced for patient care in 2008. Fospropofol has slower onset and longer duration, but can be a possible alternative to propofol. The cost difference between the two agents significantly favors propofol.

\section{Remifentanil}

A synthetic opioid agonist with potency similar to fentanyl yet metabolized by plasma esterases, remifentanil has a half-life shorter than 5 minutes. This makes remifentanil an effective opioid analgesic without the concerns of prolonged effects due to redistribution or context sensitivity. Remifentanil can be administered as a continuous infusion over the course of the procedure, with clinical effects resolving within minutes after discontinuing the infusion. It can be administered both at an analgesic or sedative level. This makes it particularly useful for the obese patient and for patients with obstructive sleep apnea. Due to a predominant $\mu$-receptor effect, remifentanil can cause analgesia, respiratory depression, depression of cardiac output, and systemic vascular resistance, as well as chest rigidity. Remifentanil should be used with caution in the elderly population and individuals with significant cardiac failure.

\section{Dexmedetomidine}

This is an $\alpha 2$-receptor agonist that has a high concentration in the locus coeruleus, where it exercises inhibitory effects inducing sleep-like anesthesia. Due to its presence in the sympathetic pre- and postsynaptic areas, it prevents the release of norepinephrine from the presynaptic storage. An $\alpha 1$-antagonist effect adds to its complex clinical effects, which include, sedation, reduction in blood pressure and heart rate, and analgesia with minimal respiratory depression. Dexmedetomidine is useful as an adjunct agent for patients who require lesser use of narcotics. A loading dose of $0.5-1.0 \mu \mathrm{g} / \mathrm{kg}$ is given over 10 minutes, and if needed an infusion of $0.2-0.7 \mu \mathrm{g} / \mathrm{kg} / \mathrm{h}$ can follow. While dexmedetomidine is useful as an adjunct agent with other anesthetics, its effect as a single-agent sedative for third molar surgery was not as impressive as when combined with a small dose of midazolam, according to Smiley and Prior. ${ }^{30}$

\section{Is total intravenous anesthesia more advantageous than inhalation anesthesia?}

Lebenbom-Mansour et al ${ }^{31}$ evaluated 60 patients undergoing orthopedic surgery who were divided into four groups: group 1, propofol induction followed by desflurane/nitrous maintenance; group 2, propofol induction followed by propofol/nitrous maintenance; group 3, desflurane/nitrous induction and maintenance; and group 4, desflurane induction and maintenance. While the quality of induction was inferior in groups 3 and 4, there was more rapid emergence in group 4 compared to the other three groups. Intermediate recovery showed no significant difference between groups 2 and 4, which were faster than groups 1 and 3. Group 3 had the most PONV incidents of all four groups.

In a multicenter study of patients undergoing knee arthroscopy, Raeder et $\mathrm{al}^{32}$ compared maintenance of anesthesia with sevoflurane versus propofol infusion; the sevoflurane group had faster recovery but increased incidence of PONV. Hemodynamic side effects were minimal in both groups.

In another study, ${ }^{33} 60$ patients undergoing laparoscopic cholecystectomy received desflurane or propofol for anesthetic maintenance; while the desflurane group woke up faster, they had a significantly higher incident of PONV and pain in the recovery phase. When comparing vital capacity induction using sevoflurane versus intravenous induction with propofol, Philip et $\mathrm{al}^{34}$ reported faster induction and higher patient satisfaction in the sevoflurane group among adults undergoing ambulatory surgery.

Tang et $\mathrm{al}^{35}$ compared two groups of spontaneously breathing patients who received either desflurane or propofol anesthesia. Patient mobility during surgery was observed more significantly in the propofol group, while desflurane recovery was significantly faster. In order to bring desflurane PONV incidence down to a comparable level to the propofol group, the desflurane group had to receive a triple PONV prophylaxis with ondansetron, droperidol, and metoclopramide.

The literature does not offer compelling evidence for the advantage of one technique versus the other in improving recovery profile and cognitive function. ${ }^{36,37}$ Further large-scale studies need to be conducted to validate either technique. This leaves the door open to a patient-specific and procedure-specific protocol with combined inhalation and intravenous techniques as promising for superior outcome. Combined intravenous techniques can also be applied utilizing infusion of propofol/ketamine, propofol/remifentanil, or propofol/dexmedetomidine.

\section{Electroencephalography-driven monitoring}

The concept of electroencephalography-driven level of consciousness monitoring has existed for several decades. In the late 1980s, the development of bispectral index monitoring ushered 
in a new era in the utilization of electroencephalography-driven depth of anesthesia monitoring, promising improved and faster extubation and recovery. ${ }^{38-42}$ Utilizing this technology shows promise in providing feedback regarding depth of anesthesia as a prelude to tracheal intubation without the use of neuromuscular blocking agents. ${ }^{43}$

\section{Ambulatory anesthesia and postoperative course of recovery}

Provision of anesthesia care includes the management of postsurgical pain and PONV. Jakobsson ${ }^{44}$ described a multimodal approach to pain management comprising lowdose narcotics, nonsteroidal anti-inflammatory drugs, oral acetaminophen, and intravenous dexamethasone.

\section{How about regional versus general anesthesia?}

A meta-analysis ${ }^{45}$ of regional versus general anesthesia did not show an advantage of one technique versus the other in reduction of surgical time. Unique circumstances regarding patientspecific history or surgical procedure may impact the decision to choose one versus the other. De Oliveria et $\mathrm{al}^{46}$ published a meta-analysis of multiple studies validating that the systemic use of magnesium can significantly reduce postoperative pain regardless of the type of anesthesia provided.

\section{PONV management}

Management of PONV is also very important in creating favorable postoperative recovery conditions. Gan et $\mathrm{al}^{47}$ recently published consensus guidelines for the management of postoperative nausea and vomiting. The consensus includes:

1. identification of patients with highest risk, particularly females, under age 50 years with previous history of PONV

2. procedures with higher risk of PONV, such as strabismus

3. minimizing the use of nitrous oxide and inhalation agents

4. minimizing the use of postoperative narcotics

5. preemptive prophylaxis with $5-\mathrm{HT}_{3}$-receptor antagonists (eg, ondansetron) and steroids (eg, dexamethasone).

\section{Accreditation of ambulatory facilities and mobile anesthesiology organizations}

The Accreditation Association for Ambulatory Health Care, as well as the Joint Commission, provide accreditation mechanism for ambulatory facilities, office-based surgical facilities, and mobile anesthesiology organizations. The American Association for Accreditation of Ambulatory Surgery Facilities provides accreditation for ambulatory surgical facilities. The value of accreditation is in creating guidelines for nationally accepted standards of care and quality improvement. It provides the organizations and providers with a framework to follow in clinical care, record keeping, benchmarking, and quality improvement. While logistical burdens might discourage some providers from voluntarily pursuing accreditation, it is very clear that many regulatory bodies and third-party reimbursement providers will be attaching compensation of care to outcome documentation and accreditation. Organizations that have pursued such a path voluntarily might have an advantage over those that will follow suit.

The Anesthesia Patient Safety Foundation (patient-outcome registry), sponsored by the American Society of Anesthesiologists, as well as the Society for Ambulatory Anesthesia Clinical Outcomes Registry, sponsored by the Society for Ambulatory Anesthesia and recently endorsed by the American Society of Dentist Anesthesiologists, provide a forum for collating safety outcomes of anesthesia care in the ambulatory setting. It would be an ambitious but worthy goal to aim for a national project to collect such data in the entire US. This could positively impact the future of ambulatory anesthesia care.

\section{Conclusion}

Ambulatory anesthesia in its different settings is safe, efficient, and cost-effective. Employing evidence-based anesthetic techniques as well as scientifically proven methods for controlling pain and PONV are essential for successful outcomes. Safety measures, such as patient-safety checklists, staff training, employment of highly trained providers, and adequate patient selection, can positively impact patient safety and satisfaction. With the continued advancement in the science and technology of anesthesia care, we can anticipate better and safer outcomes in the future for patient anesthesia care in the ambulatory setting.

\section{Disclosure}

The author reports no conflicts of interest in this work.

\section{References}

1. Fleisher LA. Ambulatory anesthesia. Anesthesiol Clin. 2014;32:XV

2. Apfelbaum JL, Cutter TW. The four Ps: place, procedure, personnel, and patient. Anesthesiol Clin. 2014;32:xvii-xxi.

3. Weiss AJ, Elixhauser A, Andrews RM. Characteristics of operating room procedures in US hospitals, 2011. 2014. Available from: http://www. hcup-us.ahrq.gov/reports/statbriefs/sb170-Operating-Room-ProceduresUnited-States-2011.jsp. Accessed October 3, 2014. 
4. Mathis MR, Naughton NN, Shanks AM, et al. Patient selection for day case-eligible surgery: identifying those at high risk for major complications. Anesthesiology. 2013;119:1310-1321.

5. Hollingsworth JM, Birkmeyer JD, Ye Z, Miller DC. Specialty-specific trends in the prevalence and distribution of outpatient surgery: implications for payment and delivery system reforms. Surg Innov. Epub March 6, 2014

6. Sheetz KH, Corona L, Cramm S, et al. Variation in ambulatory surgery utilization in Michigan. J Surg Res. 2014;189:255-261.

7. Metzner J, Kent CD. Ambulatory surgery: is the liability risk lower? Curr Opin Anaesthesiol. 2012;25(6):654-658.

8. Majholm B, Engbæk J, Barhtoldy J, et al. Is day surgery safe? A Danish multicenter study of morbidity after 57,709 day surgery procedures. Acta Anaesthesiol Scand. 2012;56:323-331.

9. Nekhendzy V, Ramaiah VK. Prevention of perioperative and anesthesiarelated complications in facial cosmetic surgery. Facial Plast Surg Clin North Am. 2013;21:559-577.

10. Dexter F, Maxbauer T, Stout C, Archbold L, Epstein RH. Relative influence on total cancelled operating room time from patients who are inpatients or outpatients preoperatively. Anesth Analg. 2014;118 1072-1080.

11. Hair B, Hussey P, Wynn B. A comparison of ambulatory perioperative times in hospitals and freestanding centers. Am J Surg. 2012;204:23-27.

12. Clark N, Schneider DF, Vrabec S, Bauer PS, Chen H, Sippel RS. Increased efficiency of endocrine procedures performed in an ambulatory operating room. J Surg Res. 2013;184:200-203.

13. Kurrek MM, Twersky RS. Office-based anesthesia: how to start an office-based practice. Anesthiol Clin. 2010;28:353-367.

14. Shapiro FE, Punwani N, Rosenberg NM, Valedon A, Twersky R, Urman RD. Office-based anesthesia: safety and outcomes. Anesth Analg. 2014;119:276-285.

15. Shapiro FE, Jani SR, Liu X, Dutton RP, Urman RD. Initial results from the National Anesthesia Clinical Outcomes Registry and overview of office-based anesthesia. Anesthesiol Clin. 2014;32:431-444.

16. Phillips BT, Wang ED, Rodman AJ, et al. Anesthesia duration as a marker for surgical complications in office-based plastic surgery. Ann Plast Surg. 2012;69:408-411.

17. Verghese C, Brimacombe J. Survey of laryngeal mask airway usage in 11,910 patients: safety and efficacy for conventional and nonconventional usage. Anesth Analg. 1996;82:129-133.

18. Lin B, Messieha ZS, Hoffman WE. Safety and efficacy of pediatric general anesthesia by laryngeal mask airway without intravenous access. Int J Clin Med. 2011;2:328-331.

19. Brimacombe J, Keller C. The ProSeal laryngeal mask airway: a randomized crossover study with the standard laryngeal mask airway in paralyzed anesthetized patients. Anesthesiology. 2000;93:104-109.

20. Howard-Quijano KJ, Huang YM, Matevosian R, Kaplan MB, Steadman RH. Video-assisted instruction improves the success rate for tracheal intubation by novices. Br J Anesth. 2008;101:568-572.

21. Nouruzi-Sedeh P, Schumann M, Groeben H. Laryngoscopy via Macintosh blade versus GlideScope: success rate and time for tracheal intubation in untrained medical personnel. Anesthesiology. 2009;110:32-37.

22. Aziz M. Videolaryngoscopy and the difficult airway. ASA Newsl. 2013;77:24-25.

23. Ahmed-Nusrath A, Tong JL, Smith JE. Pathway through the nose for nasal intubation: a comparison of three endotracheal tubes. Br JAnesth. 2008;100:269-274.

24. Prior S, Heaton J, Jantana K, Rashid RG. Parker flex-tip and standardtip endotracheal tubes: a comparison during nasotracheal intubation. Anesth Prog. 2010;57:18-24.

25. Sanuki T, Kotani J. Thermosoftening of the Parker flex-tip tracheal tube in preparation for nasotracheal intubation. Anesth Prog. 2013;60: 109-110.

26. Cravero JP, Beach M, Dodge CP, Whalen K. Emergence characteristics of sevoflurane compared to halothane in pediatric patients undergoing bilateral pressure equalization tube insertion. J Clin Anesth. 2000;12:397-401.
27. White PF, Tang J, Wender R, et al. Desflurane versus sevoflurane for maintenance of outpatient anesthesia: the effect on early versus late recovery and perioperative coughing. Anesth Analg. 2009;109:387-393.

28. Nathanson MH, Fredman B, Smith I, White P. Sevoflurane versus desflurane for outpatient anesthesia: a comparison of maintenance and recovery profiles. Anesth Analg. 1995;81:186-190.

29. Dexter F, Tinker JH. Comparisons between desflurane and isoflurane or propofol on time to following commands and time to discharge. A metaanalysis. Anesthesiology. 1995;83:77-82.

30. Smiley MK, Prior SR. Dexmedetomidine sedation with and without midazolam for third molar surgery. Anesth Prog. 2014;61:3-10.

31. Lebenbom-Mansour MH, Pandit SK, Kothary SP, Randal GI, Levy L. Desflurane versus propofol anesthesia: a comparative analysis in outpatients. Anesth Analg. 1993;76:936-941.

32. Raeder J, Gupta A, Pedersen FM. Recovery characteristics of sevoflurane or propofol based anesthesia for day-care surgery. Acta Anaesthesiol Scand. 1997;41:988-994.

33. Raeder JC, Mjåland O, Aasbø V, Grøgaard B, Buanes T. Desflurane versus propofol maintenance for outpatient laparoscopic cholecystectomy. Acta Anaesthesiol Scand. 1998;42:106-110.

34. Philip BK, Lombard LL, Roaf ER, Drager LR, Calalang I, Philip JH. Comparison of vital capacity induction with sevoflurane to intravenous induction with propofol for adult ambulatory anesthesia. Anesth Analg. 1999;89:623-627.

35. Tang J, White PF, Wender RH, et al. Fast-track office-based anesthesia: a comparison of propofol versus desflurane with antiemetic prophylaxis in spontaneously breathing patients. Anesth Analg. 2001;92:95-99.

36. Lindqvist M, Schening A, Granstrom A, Bjorne H, Jakobsson JG. Cognitive recovery after ambulatory anesthesia based on desflurane or propofol: a prospective randomised study. Acta Anaesthesiol Scand. 2014;58:1111-1120.

37. Ortiz AC, Atallah AN, Matos D, Da Silava E. Intravenous versus inhalational anaesthesia for pediatric outpatient surgery. Cochrane Database Syst Rev. 2014;2:CD009015.

38. Rosow C, Manberg PJ. Bispectral index monitoring. Anesthesiol Clin North America. 1998;2:89-107.

39. Gan TJ, Glas PS, Windsor A, et al. Bispectral index monitoring allows faster emergence and improved recovery from propofol, alfentanil and nitrous oxide anesthesia. Anesthesiology. 1997;87:808-815.

40. Glass PS, Bloom M, Kearse L, Rosow C, Sebel P, Manberg. Bispectral analysis measures sedation and memory effects of propofol, midazolam, isoflurane and alfentanil in healthy volunteers. Anesthesiology. 1997;86:836-847.

41. Messieha ZS, Ananda RC, Hoffman WE, Punwani IC, Koenig HM. Bispectral index system (BIS) monitoring reduces time to extubation and discharge in children requiring oral presedation and general anesthesia for outpatient dental rehabilitation. Pediatr Dent. 2005;27:500-504.

42. Messieha ZS, Ananda RC, Hoffman WE, Punwani IC, Koenig HM. Bispectral index index system (BIS) monitoring reduces time to discharge in children requiring intramuscular sedation and general anesthesia for outpatient dental rehabilitation. Pediatr Dent. 2004;26: 256-260.

43. Messieha ZS, Guirguis S, Hanna S. Bispectral index monitoring (BIS) as a guide for intubation without neuromuscular blockade in office-based pediatric general anesthesia: a retrospective evaluation. Anesth Analg. 2011;58:3-7.

44. Jakobsson JG. Pain management in ambulatory surgery - a review. Pharmaceuticals (Basel). 2014;7:850-865.

45. Liu SS, Strodtbeck WM, Richman JM, Wu CL. A comparison of regional versus general anesthesia for ambulatory anesthesia: a meta-analysis of randomized controlled trials. Anesth Analg. 2005;101:1634-1642.

46. De Oliveria GS, Castro-Alves LJ, Khan JH, McCarthy RJ. Perioperative systemic magnesium to minimize postoperative pain: a meta-analysis of randomized controlled trials. Anesthesiology. 2013;119:178-190.

47. Gan TJ, Diemunsch P, Habib AS, et al. Consensus guidelines for the management of postoperative nausea and vomiting. Anesth Analg. 2014;118:85-113. 


\section{Publish your work in this journal}

Ambulatory Anesthesia is an international, peer reviewed, open access journal publishing articles that address all aspects of ambulatory anesthesia practice, in particular: anesthetic techniques, sedation and safety practices, pharmacokinetics, preoperative evaluation, analgesia interventions, regulatory and compliance issues, postoperative recovery,

Submit your manuscript here: http://www.dovepress.com/ambulatory-anesthesia-journal patient satisfaction, administrative topics, and cost analysis themes. The manuscript management system is completely online and includes a very quick and fair peer review system, which is all easy to use. Visit http://www.dovepress.com/testimonials.php to read real quotes from published authors. 